\title{
The Smart Grid as a public health protection tool: using Smart Grid technologies for monitoring distribution systems
}

\author{
G. S. Symmonds \& T. T. Hill \\ Global Water Resources, Phoenix, Arizona, USA
}

\begin{abstract}
Since the introduction of the Safe Drinking Water Act, there has been a marked reduction in the number of water-borne disease outbreaks attributed to water treatment systems. Over the same period, however, the percentage of disease outbreaks attributable to defects in the distribution system has increased exponentially. Interestingly, as a result of the continuous aging infrastructure employed by our water utilities, the number of waterborne disease cases has increased in the last decade. The smart grid for water can be used to significantly improve both the utility's understanding of water quality in the distribution system, but can dramatically increase the response time and provide the means to ensure public health protection. This paper will present the use of the Analytical Water Quality Assurance Program developed to provide instantaneous water quality notifications throughout the utility organization.
\end{abstract}

Keywords: Smart Grid for water, public health protection, distribution systems.

\section{Introduction}

In 1974, the Safe Drinking Water Act (SDWA) was enacted, providing a cohesive federal set of standards for water quality delivered to consumers. As a result of the SDWA (and amendments in 1986 and 1996), utilities were required to meet Maximum Contaminant Levels (MCLs) for certain constituents prior to water entering the distribution point. Today, the SDWA regulates 87 primary contaminants and the USEPA publishes and maintains a Contaminant Candidate List and is required to make regulatory determinations (ie a decision to regulate or not) for at least five potential contaminants every five years (USEPA has 
recently published Contaminant Candidate List 3 including 12 microbial contaminants and 106 chemical contaminants).

Notably, however, the majority of these contaminants are regulated at the Entry Point to the Distribution System (EPDS) and not in the distribution system. In fact, only three of the SDWA rules require monitoring in the distribution system: the Lead and Copper Rule, the Total Coliform Rule, and the Disinfectant and Disinfection By-Products Rule. The result is that there are few regulatory requirements to monitor for water degradation in the distribution system, and indeed the proliferation and impact of contamination can be at a timescale several orders of magnitude faster than the requirement to monitor. This is particularly true of microbial contamination where the rapid proliferation of microbes and their ability to reproduce can result in widespread illnesses and deaths (figure 1).

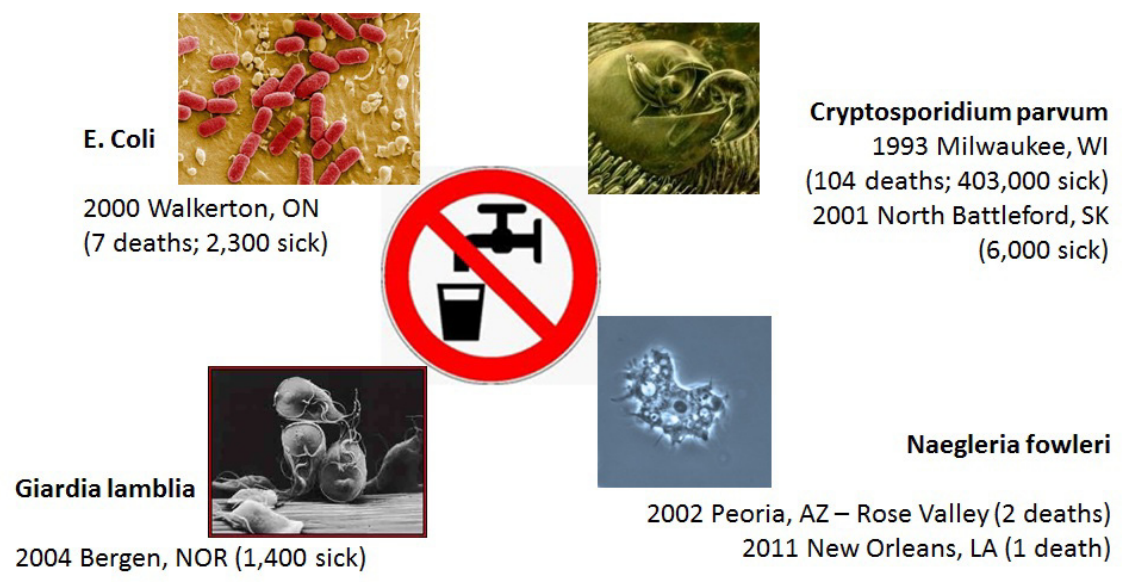

Figure 1: $\quad$ Waterborne disease outbreaks.

\section{Distribution systems and public health}

Distribution systems remain a vast array of potential problems for utilities. A typical utility has approximately 80 to 100 feet of transmission and distribution mains per connection. A utility serving 15,000 connections can have over 200 miles of distribution main. And the volume of water contained in that distribution system can rival the actual tank storage employed by the utility. For instance, Global Water - Santa Cruz Water Company, a utility serving approximately 45,000 people (17,000 connections) in Arizona, has 1.2 million feet of transmission and distribution main with a total volume of over 5 million gallons. This un-monitored and by definition, distributed, storage system is a great liability for utilities. 
Despite the introduction of standards, rules and regulations through the SDWA, there has been a marked increase in the average annual number of reported cases of water-borne disease (figure 2).

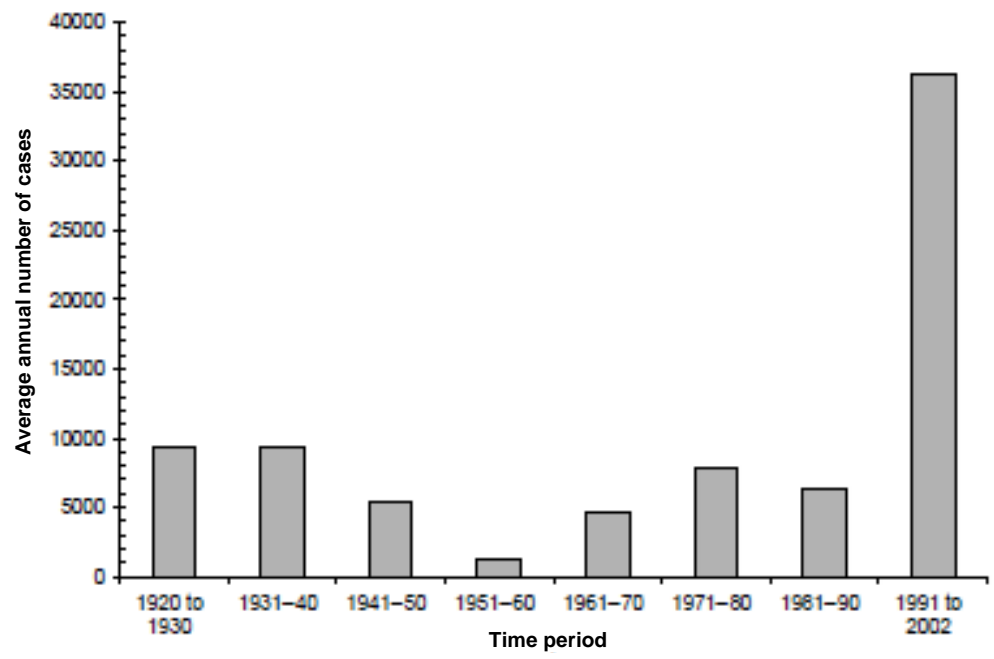

Figure 2: Average annual number of cases of health effects from water sources [1].

The cause of the increasing number of cases of waterborne illness in the United States may be related to the fact that our distribution infrastructure is at, or in many cases, beyond its design life. The introduction of the SDWA and the regulatory requirements to ensure safe water entering the distribution system resulted in dramatic and sustained decreases in the percentage of these illness cases that could be attributed to water treatment. However, due to the age of our distribution systems, and the lack of comprehensive regulatory oversight of potential water quality degradation within these distributed storage systems, the percentage of waterborne illness causes attributable to the distribution system has increased exponentially (figure 3 ).

US water systems experience 240,000 water main breaks annually, resulting in the loss of 1.7 trillion gallons of water [2]. The USEPA reports that large utility breaks in the Midwest increased from 250 per year to 2,200 per year during a 19-year period demonstrating that the number of water main breaches is increasing as our infrastructure nears the end of the its service life. In 2003, the City of Baltimore, Maryland, reported 1,190 water main breaks - an average of more than three per day.

But it is not only the integrity of our water systems that cause concern. Our wastewater infrastructure is also deteriorating and resulting in cross contamination. In the US, 3-10 billion gallons of untreated wastewater are discharged annually. These "sanitary sewer overflows" as a result of blockages 


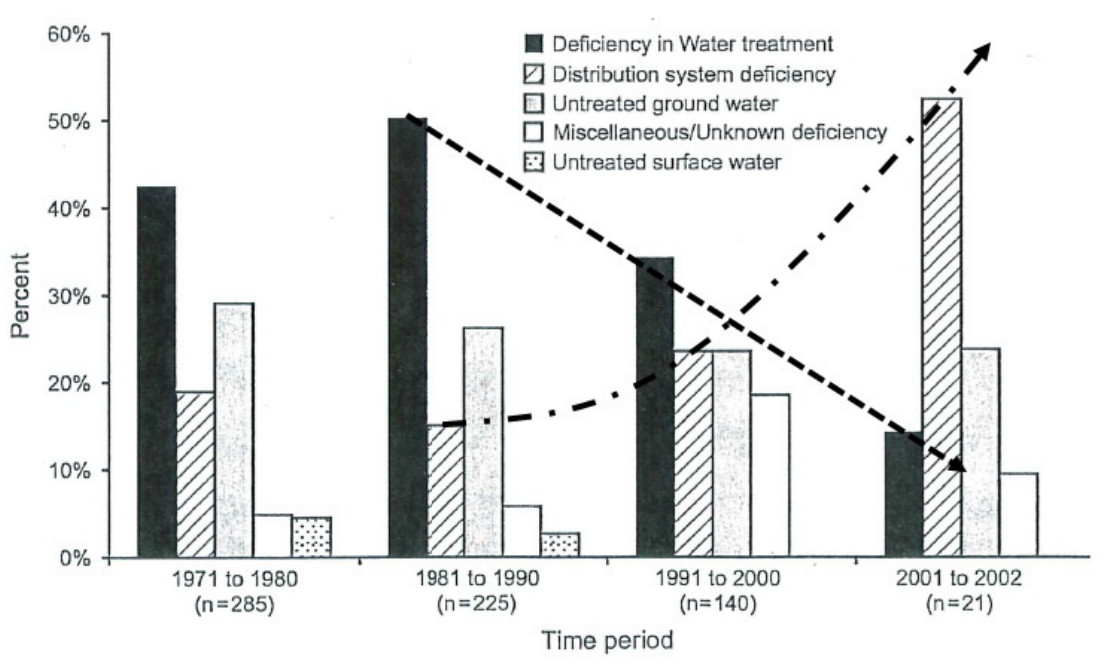

Figure 3: Sources of waterborne disease outbreaks shifting to distribution systems [1].

or wastewater main breaks can have direct and immediate impacts on public health [2]:

- In 1989, sanitary sewer overflows in Cabool, Missouri, contaminated drinking water distribution lines, causing 243 cases of diarrhoea and 4 deaths.

- In 1993, direct contact with a discharge of untreated sewage in Ocoee, Florida, resulted in 39 cases of hepatitis A.

This trend is likely to continue upwards as monitoring water quality and physical condition of distribution systems is extremely difficult, and our infrastructure is continuously aging.

There is a very strong association of water-borne disease outbreaks with reporting of loss of pressure related to burst water mains [3]. The fact is that the conditions under which repairs are made are far less sanitary than those of new construction, resulting in a high probability of contamination from the surrounding materials. A study of the water/soil matrix surrounding water mains noted that faecal coliform bacteria were detected in $43 \%$ of water samples and $50 \%$ of the soil samples. $56 \%$ of these samples were also positive for viruses [4].

Based on these conditions, and the overall emergency requirement to return water service as quickly as possible to residents, it is not unexpected that there can be contamination introduced during these repairs.

Another aspect of the distribution system that is often overlooked is the potential for backflow conditions. Backflow will occur when the water pressure in the public water supply is lost, reduced, or if the customer's water pressure becomes greater than the public supply. While most utilities employ backflow 
programs and require backflow prevention devices to be installed at locations where there is a known or suspected risk due to the type of customer or where the local hydraulic conditions warrant, there remains the probability that routine backflow can occur - and from sources that the utility may not fully understand, or from cross connections that may exist that are not known to the utility.

\section{Invisible infrastructure}

Water and sewer infrastructure suffer from invisibility. The infrastructure is in the ground, non-hazardous (compared to natural gas and power) and decidedly low-tech. Further, much of the infrastructure was installed not by city or utility crews, but by developers and land owners. It is not surprising then that most utility departments - unless they have been judicious about requiring and maintaining record drawings and as-builts - have very little knowledge of the location of their systems. And field crews typically do not have direct access to vital records such as piping material, size, installation dates, etc. which severely limits their ability to respond to both emergencies and routine calls.

This lack of direct infrastructure knowledge, the lack of direct monitoring of water quality degradation in distribution systems, and the fact that much of our water and wastewater infrastructure was installed generations ago and is reaching the end of its useful life, are enabling conditions that can result in catastrophic failures, and potentially impact the health of a significant number of people.

In order to address these issues, broad-scale infrastructure replacement is required. The US Government Accountability Office has estimated the costs of this replacement to be in the order of $\$ 300$ billion to $\$ 1$ trillion dollars [5]. Neglecting this issue will accelerate an already monumental infrastructure degradation problem. The U.S. Geological Survey estimates that water lost from U.S. water distribution systems is 1.7 trillion gallons per year at a national cost of $\$ 2.6$ billion per year [2]. This degrading infrastructure - unless managed effectively through structured monitoring and replacement programs - represents a significant threat to our public health mandate.

Clearly, the financial condition of many municipalities precludes immediate investment in large-scale infrastructure replacement. A means of efficiently managing infrastructure while at the same time increasing vigilance of water quality in distribution systems is required. The Smart Grid for Water can achieve these objectives, and allow for municipalities to maximize the efficiency of their capital program by identifying where the most critical areas are and directing operational resources to assure public health protection.

\section{Using the Smart Grid to improve public health}

The Smart Grid for Water can be used to significantly improve not only the utility's understanding of water quality in the distribution system, but can dramatically decrease response times and provide the means to ensure public health protection, by improving the temporal and spatial quality of the data. 
For example, by combining customer input via call centres, highly granular consumption data from the Customer Information System, operational information from Supervisory Control and Data Acquisition (SCADA) systems, hydraulic modelling data and geo-referenced spatial asset management data, a rapid, visual identification of water distribution system health can be built allowing operations staff to immediately respond to any potential issue. Further, leak detection flags and reverse flow flags from Advanced Metering Infrastructure (AMI) metering systems can be employed to both identify potential ingress of contaminants, and where hydraulic conditions exist that promote reverse flows.

The Smart Grid for Water also provides immediate and detailed access to information in the case of natural disasters which can directly safeguard human health and provide for more immediate response and recovery. On 11 May 2011, a magnitude 5.1 earthquake struck Lorca, Spain causing significant structural damage. The local water utility was able to use some facets of the Smart Grid for Water - data integration, electronic metering and communications - to quickly identify and rectify the impacts on the water distribution system [6]:

- $\quad$ Identification of 5 major leaks in the distribution system

- Identification of 22 leaks inside buildings

- $\quad$ Re-configuration of water distribution system to recover service

\section{Decision support systems}

Using the tools of the Smart Grid for Water, a Decision Support System known as the Analytical Water Quality Assurance (AWQuA) Program facilitating the monitoring of the distribution system is under development (figure 4).

The AWQuA Program aggregates customer water quality/aesthetic issues (CIS) with operational data (SCADA), compliance data (LIMS), flow data (AMI), maintenance data (Asset Management) and engineering data (hydraulic models) to determine the likelihood, extent and impact of a potential distribution system water quality issue. The AWQuA Program allows for early detection and classification of any potential public health issue and proactively allows operations staff to identify the necessary rectification plan, while simultaneously allowing compliance staff to notify regulatory agencies. It also allows for automatic consumer notification via Interactive Voice Response (IVR) systems, Reverse 911 calling or text messaging. With a rich information system, customer service staff can provide up-to-date information to consumers who may call in.

The AWQuA Program also serves as an infrastructure replacement trigger by identifying the most critical areas - from a public health protection and infrastructure reliability perspective.

The most important aspect of the AWQuA Program is the "push" of information to operations, engineering and compliance staff in order to accelerate investigation and rectification processes. 


\section{Analytical Water Quality Assurance Program}

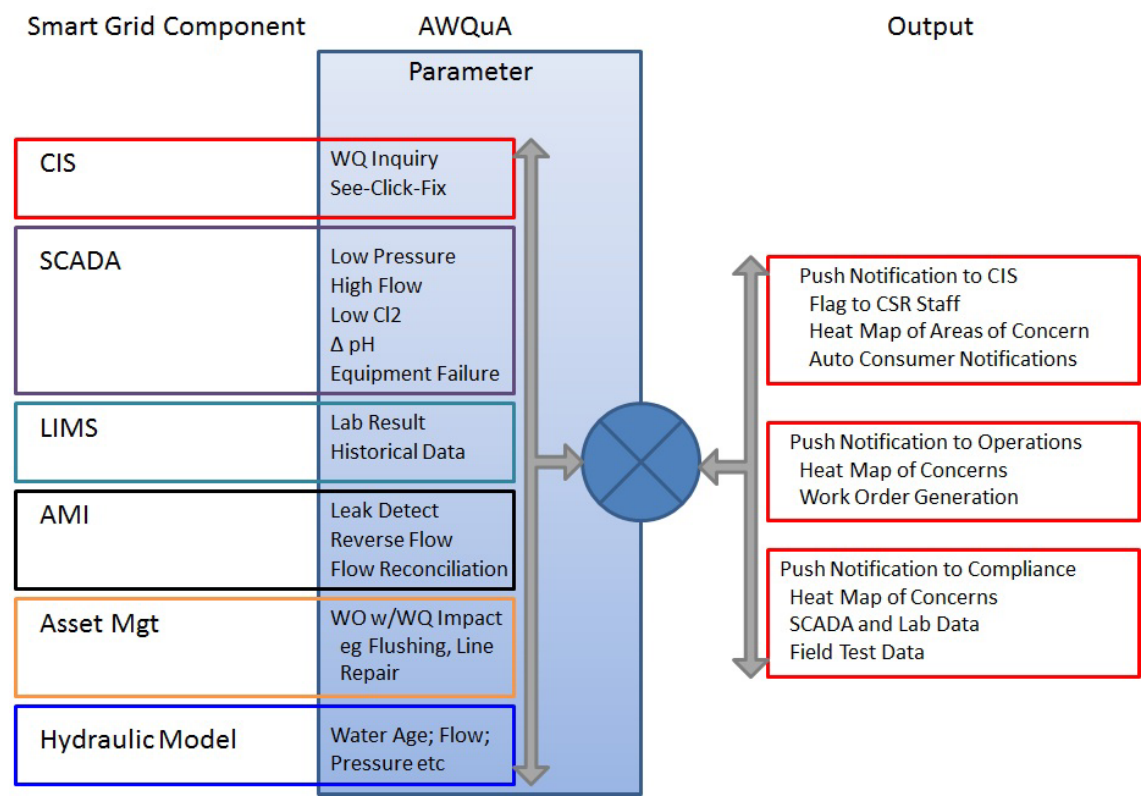

Figure 4: The AWQuA Program.

AWQuA Program integrates several major data systems into an information system. It does this by combining information from consumer water quality complaints (CIS), asset management (CMMS), SCADA, AMI, LIMS and engineering systems (hydraulic models etc.) Each provides a specific benefit for monitoring distribution systems.

\subsection{CIS - Consumer water quality complaint assessments}

An often overlooked source for water quality monitoring is the utility's customers: "Feedback from drinking water consumers is particularly valuable to water suppliers, because it is a 'real-time' water quality assessment at no cost to the utility. Additionally, these water quality monitors are located at every point in the distribution system where water is being used at all times.” [7].

Consumers are the first line of defence in water quality monitoring. Unfortunately, their observations are often misdiagnosed or mis-categorized due to the non-technical nature of both the consumer and the lack of water quality knowledge available to customer service representative (CSR) who receives the complaint. In addition, in many cases, the information the CSR has relates only to that call - he or she lacks the geographic and temporal relationships between calls.

The AWQuA Program provides an instant geographic relationship with other consumer complaints (figure 5), allowing the CSR to provide the consumer with 
up to date information regarding any potential problems in the area. By sharing information with asset management and SCADA systems, a more complete response to the consumer can be made.

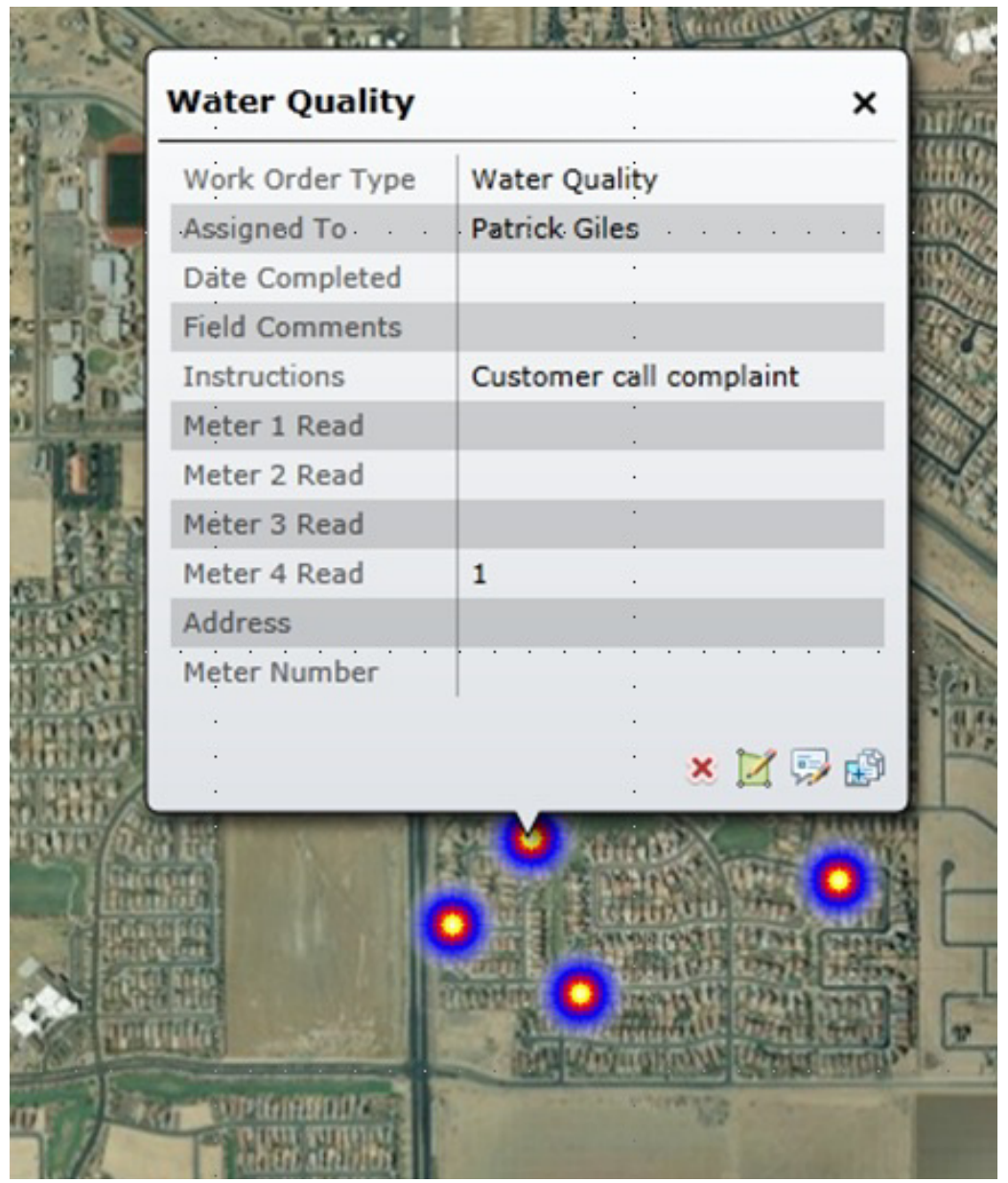

Figure 5: Geographic relationship of water quality complaints.

A direct benefit of using an integrated information platform for water quality monitoring for distribution systems is the ability to use functions like SeeClickFix (www.seeclickfix.com) to accelerate information transfer.

\subsection{Asset management}

An oft cited quote in quality management is that you cannot manage what you do not measure. In the water business, you can't manage what you do not know is there. 
Many utilities still rely on quarter-section maps, marked up with hand-written notes to identify infrastructure (or worse on the corporate knowledge of specific individuals). Not only is this an inefficient data repository, it limits the availability of that data to one person. To be successful, the 21st century water utility must become a “data gateway”, eliminate data gatekeepers, and maximize the potential uses for data.

In order to address water quality in distribution systems, detailed information on the following maintenance activities is required. For example [7]:

$\begin{array}{ll}\text { - } & \text { Start-up or shutdown of treatment proce } \\ \text { - } & \text { Changes in treatment processes. } \\ \text { - } & \text { Water main breaks. } \\ \text { - } & \text { Sanitary sewer overflows } \\ \text { - } & \text { Sewer main breaks } \\ \text { - } & \text { Fire fighting activities. } \\ \text { - } & \text { Distribution system flushing activities. } \\ \text { - } & \text { Construction near waterlines. }\end{array}$

This information is available to staff through the AWQuA Program through links with the Asset Management system.

\subsection{AMI systems}

Advanced Metering Infrastructure (AMI) systems not only accurately read meters at a dramatically increased frequency, but also assist in finding and controlling leaks. Reducing non-revenue water (the difference between water pumped, treated, and supplied to the distribution system versus water that actually reaches customers) is of critical importance in maintaining public health as it is an indication of a route of potential contaminant ingress.

Leak detection flags (at consumer meters), reverse flow indicators and inconsistencies between pumped versus billed volumes are all essential information in the assessment of distribution system health.

\subsection{SCADA/Hydraulic modelling}

Knowing the dynamic regime in which distribution systems operate is critical for understanding the potential water quality implications of events to determine the proper course of action.

Push reports on high flow incidents (fires etc), low pressure incidents (main breaks etc) allow operations, customer service and compliance staff to assess the operational status of the distribution system, and to project any water quality impacts.

\subsection{Laboratory systems}

Laboratory Information Management Systems (LIMS) contain current and historical water quality data for both treatment and distribution systems. Access 
to this data facilitates water quality issue rectification, but can also be used to validate and assess past events as models for future events.

\section{Conclusions}

The number of waterborne illness incidents is increasing - as result of degrading infrastructure and the fact that much of our infrastructure is uncovered to a large extent by the regulatory scheme, and monitored by the utility at a timescale that is insufficient to counter the rapid spread of contaminants.

Routine maintenance, emergency repairs, backflow conditions and other issues have shifted the source of contamination from treatment systems or source water to distribution systems. Unfortunately, utilities today lack the comprehensive monitoring programs that are employed on the treatment side of water delivery. That is driven by the fact that while treatment systems can be effectively monitored by one system - SCADA - distribution system monitoring requires the integration of many more information systems - CIS, LIMS, AMI, Asset Management etc.

Most utilities lack the data and system-level integrations necessary to combine the different types of data (physical, customers, flow, pressure, lab, etc.) to make system determinations or to perform system-wide diagnostics.

The Smart Grid for Water changes that, and provides the tools to make quantitative determinations on the quality of water in the distribution system, and ensuring public health, while maximizing the efficiency of maintenance and capital expenditure budgets.

\section{References}

[1] Craun, M.F., et al., Waterborne outbreaks reported in the United States, J. Wat. Health 4(Suppl. 2), 19-30, 2006.

[2] USEPA, “Addressing the Challenge Through INNOVATION”, Office of Research and Development National Risk Management Research Laboratory, 2007.

[3] Hunter. P.R., et al., Self-Reported Diarrhea in a Control Group: A Strong Association with Reporting of Low-Pressure Events in Tap Water, Clinical Infectious Diseases 2005; 40:e32-4.

[4] Karim, Abbaszadegan, LeChevallier "Potential for pathogen intrusion during pressure transients”. J of American Water Works Association 2003:95:134-46.

[5] GAO Report GAO-02-764 "WATER INFRASTRUCTURE - Information on Financing, Capital Planning, and Privatization”, August 2002

[6] Molina, M., "Full scale application of network monitoring tools for leakage reduction and asset rehabilitation prioritization". Presentation to Smart Water Networks Forum, Paris, 18 May 2011.

[7] U.S. Army Center for Health Promotion and Preventive Medicine, "USACHPPM TG 284 Drinking Water Consumer Complaints: Indicators from Distribution System Sentinels”, May 2003. 